\title{
Efeitos da utilização do bundle na prevenção de pneumonia associada à ventilação mecânica: revisão integrativa
}

\author{
Effects of the Use of Care Bundles in Preventing Ventilator-Associated \\ Pneumonia: An Integrative Literature Review \\ Una revisión integrativa de los efectos del uso de medidas para la \\ prevención de neumonía asociada a ventilación mecánica
}

Como citar este artigo: Maran, Edilaine; Spigolon, Dandara Novakowski; Matsuda, Laura Misue; Teston, Elen Ferraz; Oliveira, João Lucas Campos de; Souza, Verusca Soares de; Marcon, Sonia Silva. Efeitos da utilização do bundle na prevenção de pneumonia associada à ventilação mecânica: revisão integrativa Revista Cuidarte. 2021;12(1):e1110. http://dx.doi.org/10.15649/cuidarte.1110

\section{Revista Cuidarte \\ Rev Cuid. Ene. - Abril. 2021; 12(1): e1110 \\ doi) $h$ ttp://dx.doi.org/10.15649/cuidarte.1110}

E-ISSN: 2346-3414

(1) Edilaine Maran

(1) Dandara Novakowski Spigolon²

(1) Laura Misue Matsuda ${ }^{3}$

(1) Elen Ferraz Teston ${ }^{4}$

(1) João Lucas Campos de Oliveira ${ }^{5}$

(1) Verusca Soares de Souza 6

(1) Sonia Silva Marcon ${ }^{7}$

1 Universidade Estadual do Paraná - Paranavaí (PR), Brasil. E-mail: edi_enf@hotmail.com Autor Correspondente

2 Universidade Estadual do Paraná - Paranavaí (PR), Brasil. E-mail:

dandaraspigolon@gmail.com

3 Universidade Estadual de Maringá - Maringá (PR), Brasil. E-mail:

lauramisuem@gmail.com

4 Universidade Federal de Mato Grosso do Sul - Campo Grande (MS), Brasil. E-mail: elen-1208@hotmail.com

5 Universidade Federal do Rio Grande do Sul - Porto Alegre (RS), Brasil. E-mail: joaolucascampos@hotmail.com

6 Universidade Federal de Mato Grosso do Sul - Coxim (MS), Brasil. E-mail: veruscasoares@gmail.com

7 Universidade Estadual de Maringá - Maringá (PR), Brasil. E-mail:

soniasilva.marcon@gmail.com

\section{Resumo}

Introdução: A Pneumonia Associada à Ventilação Mecânica é uma infecção relacionada à assistência à saúde que predomina em unidades de terapia intensiva e que compromete a segurança do paciente. Objetivo: Identificar na literatura científica os efeitos do uso de Bundles na prevenção da Pneumonia Associada à Ventilação Mecânica em Unidade de Terapia Intensiva. Método: Revisão integrativa realizada com estudos disponibilizados nas bases dados online: Cumulative Index to Nursing and Allied Health Literature, Cocrhane, Literatura LatinoAmericana e do Caribe em Ciências da Saúde, Medical Literature Analysis and Retrieval System Online, Scientific Electronic Library Online e Scopus. A busca dos estudos nas bases de dados se deu no período de dois de junho a 18 de julho de 2018, no idioma inglês ou português, sem limite de tempo de publicação, utilizando os termos dos DeCS e MeSH. O recrutamento foi realizado por dois pesquisadores independentes. Dos artigos selecionados, extraíram-se as informações: ano, país de origem, objetivo(s), grau de recomendação científica e tipo de estudo, intervenção, conformidade do uso do Bundle, principais resultados e conclusões. Resultados: Dentre as 20 publicações analisadas, 13 eram de diferentes países e a maioria dos estudos $(n=18)$ constata que o uso de Blundes reduziu significativamente a taxa de Pneumonia Associada à Ventilação Mecânica, com impacto na redução da mortalidade, no tempo de internação e nos custos hospitalares. Os principais componentes de escolha para composição do pacote de cuidado foram à elevação da cabeceira e o protocolo de higiene oral. Conclusão: Apesar de existir espaço para estudos com maior grau de recomendação, o rol de evidências de muitos países indica que o uso de Bundles tem efeito positivo na prevenção da pneumonia associada a ventilação mecânica e também na melhoria de desempenho institucional, pela redução de custos hospitalares.

Palavras chave: Pacotes de Assistência ao Paciente; Pneumonia Associada à Ventilação Mecânica; Segurança do Paciente; Unidades de Terapia Intensiva.

Recebido: dezembro 30 de 2019

Aceito: julho 10 de 2020

Publicado: novembro 13 de 2020

$\square *$ Correspondencia

Edilaine Maran

E-mail:edi_enf@hotmail.com 


\title{
Effects of the Use of Care Bundles in Preventing Ventilator-Associated Pneumonia: An Integrative Literature Review
}

\begin{abstract}
Introduction: The ventilator-associated pneumonia is an infection related to health care that predominates in intensive care units and compromises patient safety. Objective: To identify the effects of care bundles in preventing ventilator-associated pneumonia in the Intensive Care Unit (ICU). Materials and Methods: An integrative literature review was conducted based on the studies available in the online databases Cumulative Index to Nursing and Allied Health Literature, Cochrane Library, Latin American and Caribbean Health Sciences Literature, Medical Literature Analysis and Retrieval System Online, Scientific Electronic Library Online and Scopus. Articles were selected from these databases between June 2 and July 18, 2018 in English and Portuguese without any publication time limit using DeCS and MeSh descriptors. Two independent researchers carried out the article selection process, from which information related to publication year, country of origin, objective(s), degree of scientific recommendation and type of study, intervention, compliance with the use of care bundles, main results and conclusions was collected. Results: A total of 13 out of 20 publications analyzed were from different countries and in most studies $(n=18)$ the use of care bundles was found to significantly reduce the incidence of ventilator-associated pneumonia, resulting in a reduction in mortality, length of hospital stay and costs. The main components chosen to put together care bundles included head of bed elevation and oral care. Conclusion: Although studies with a higher degree of recommendation are possible, data from several countries suggest that the use of care bundles had a positive effect on ventilator-associated pneumonia, as well as on improving institutional performance by reducing health care costs.
\end{abstract}

Key words: Patient Care Bundles; Pneumonia, Ventilator-Associated; Patient Safety; Intensive Care Units.

\section{Una revisión integrativa de los efectos del uso de medidas para la prevención de neumonía asociada a ventilación mecánica}

\section{Resumen}

Introducción: La neumonía asociada a la ventilación mecánica es una infección relacionada con el cuidado de la salud que predomina en las unidades de cuidados intensivos y que compromete la seguridad del paciente. Objetivo: Identificar en la literatura científica los efectos del uso de medidas para la prevención de la neumonía asociada a ventilación mecánica en Unidades de Cuidados Intensivos. Materiales y métodos: Se llevó a cabo una revisión integrativa con los estudios disponibles en las bases de datos en línea Cumulative Index to Nursing and Allied Health Literature, Biblioteca Cochrane, Literatura Latinoamericana y del Caribe en Ciencias de la Salud, Medical Literature Analysis and Retrieval System Online, Scientific Electronic Library Online y Scopus. La búsqueda de artículos se realizó en estas bases de datos entre el 2 de junio y el 18 de julio de 2018 en inglés y portugués sin límite de tiempo de publicación a través de los descriptores DeCS y MeSH. Dos investigadores independientes llevaron a cabo el proceso de selección de artículos, de los que se extrajo la información relacionada con el año, país de origen, objetivo(s), grado de recomendación científica y tipo de estudio, intervención, conformidad del uso de medidas de prevención, principales resultados y conclusiones. Resultados: De las 20 publicaciones analizadas, 13 eran de diferentes países y en la mayoría de los estudios $(n=18)$ se encontró que el uso de las medidas preventivas redujo significativamente la incidencia de neumonía asociada a ventilación mecánica, con un impacto en la reducción de la mortalidad, la duración de la estancia y costos hospitalarios. Los principales componentes elegidos para armar el paquete de medidas de prevención fueron la elevación de la cabeza y el protocolo de higiene bucodental. Conclusión: Aunque existe la posibilidad de realizar estudios con un mayor grado de recomendación, los datos obtenidos en varios países revelan que el uso de las medidas de prevención tiene un efecto positivo en la neumonía asociada a la ventilación, así como en la mejora del desempeño a nivel institucional, ya que reduce los costos hospitalarios.

Palabras-clave: Paquetes de Atención al Paciente; Neumonía Asociada al Ventilador; Seguridad del Paciente; Unidades de Cuidados Intensivos. 


\section{Introdução}

A Pneumonia Associada à Ventilação Mecânica (PAV) é o evento adverso (EA) predominante, relacionado à infecção nosocomial, em Unidade de Terapia Intensiva (UTI) ${ }^{1}$. De acordo com a literatura ${ }^{1,2}$, a PAV apresenta prevalência global de 15,6\%, bem como 13,5\% nos Estados Unidos; 19,4\% na Europa; 13,8\% na América Latina e 16,0\% na Ásia-Pacífico.

No cenário mundial, a PAV é considerada como um dos efeitos indesejáveis mais preocupantes em UTI, por causar danos potenciais na saúde dos indivíduos acometidos por essa complicação e também na sustentabilidade organizacional, devido ao aumento na morbidade e mortalidade, no tempo de internação e nos custos hospitalares ${ }^{3-5}$. Com isso, a PAV é considerada como infecção relacionada à assistência à saúde (IRAS) que compromete a segurança do paciente 3 .

Por se tratar de condição potencialmente prevenível, a incidência da PAV é um importante indicador de qualidade, já que permite mensurar a inconformidade entre a assistência prestada e o cuidado ideal que, em sua essência, deve ser seguro ${ }^{6}$. Apesar da sua importância, existem dificuldades para comparar a incidência de PAV, em especial envolvendo estabelecimentos de saúde diferentes porque, mesmo norteando-se por critérios rigorosos, a interpretação de alguns fatores organizacionais e estruturais pode ser subjetiva ${ }^{6}$.
Por se tratar de condição potencialmente prevenível, a incidência da PAV é um importante indicador de qualidade, já que permite mensurar a inconformidade entre a assistência prestada e o cuidado ideal que, em sua essência, deve ser seguro

Embora a PAV se constitua em infecção grave, ela pode ser considerada um EA evitável pela atenção cautelosa no processo de assistência7. Em virtude da complexidade das ações preventivas da PAV, o uso de bundles, conhecidos como pacotes de cuidados ou de intervenções baseados em evidências, têm sido preconizados ${ }^{3,8}$. Isso porque, desde que implementados de forma racional, o seu uso se constitui em suporte importante para a segurança do paciente 9 .

Ainda que se reconheça os benefícios do uso dos bundles na prevenção de PAV, não há consenso na literatura acerca das medidas básicas para a sua composição. Com isso, a verificação continua dos reais efeitos dos conjuntos de práticas realizadas por diferentes equipes é uma estratégia que coaduna com as proposições de melhoria contínua dos cuidados em saúde.

Cabe salientar que, embora tenha obtido avanços, o setor saúde enfrenta diversos desafios relacionados à segurança do paciente ${ }^{10}$. Com isso, faz-se importante que a equipe multiprofissional promova medidas e estratégias que assegurem o monitoramento adequado dos desfechos de saúde e o bem-estar dos pacientes críticos, com vistas à prevenção de EAs ${ }^{11}$.

Na perspectiva de versar sobre cuidado pautado na segurança do paciente em ventilação mecânica, faz-se necessário investigar os benefícios da utilização dos bundles no contexto da segurança do paciente e; por conseguinte, na prevenção da PAV. Deste modo, a identificação de como a literatura científica tem abordado a temática é importante porque, a partir disso, é possível elaborar novas estratégias voltadas à redução de riscos em saúde e à melhoria da qualidade da assistencial. 
Mediante ao exposto, o presente estudo tem como objetivo identificar na literatura científica os efeitos do uso-de Bundles na prevenção da PAV em UTI.

\section{Materiales y Métodos}

Revisão integrativa que seguiu as diretrizes estabelecidas pelo Preferred Reporting Items for Systematic Reviews and Meta-Analyses (PRISMA) ${ }^{12,13}$ e percorreu cinco etapas: elaboração da questão de pesquisa (identificação do problema), busca na literatura dos estudos primários, avaliação dos estudos primários, análise dos dados e apresentação da revisão ${ }^{14}$.

Como questão de pesquisa estabeleceu-se:"Quais são os efeitos do uso de Bundles na prevenção da PAV em UTI?" Para orientar a elaboração da pergunta norteadora desta Revisão e a busca na literatura, foi utilizada a estratégia do acrônimo PICO $^{15}$ (população, intervenção, comparação, resultados/desfechos e tipo de estudo), conforme consta no Quadro 1.

\section{Quadro 1. Elementos da estratégia PICO. Brasil, 2019 ${ }^{(15)}$}

\begin{tabular}{ll}
\hline \multicolumn{1}{c}{ Componente } & \multicolumn{1}{c}{ Definição } \\
\hline P: População de interesse & Pacientes em ventilação mecânica em UTI \\
I: Intervenção & Uso de bundle de prevenção de PAV \\
C: Comparação & Cuidado usual \\
O: Resultados/desfecho & Efeitos do bundle na prevenção da PAV \\
\hline
\end{tabular}

A busca nas bases de dados ocorreu mediante consulta aos Descritores em Ciências da Saúde (DECS) e seus correspondentes no Medical Subject Headings (MeSH). A estratégia de combinação dos descritores utilizados na busca de artigos científicos foram: 1) Patient Safety AND Pneumonia, Ventilator-Associated; 2) Patient Safety AND Pneumonia, Ventilator-Associated AND Patient Care Bundles; 3) Patient Safety AND Pneumonia, Ventilator-Associated OR Patient Care Bundles; 4) Pneumonia, Ventilator-Associated AND Patient Care Bundles; 5) Intensive Care Units AND Pneumonia, Ventilator-Associated AND Patient Care Bundles.

As bases de dados online consultadas, no período de dois de junho a 18 de julho de 2018, foram: Cumulative Index to Nursing and Allied Health Literature (CINAHL), Cocrhane, Literatura LatinoAmericana e do Caribe em Ciências da Saúde (LILACS), Medical Literature Analysis and Retrieval System Online (MEDLINE), Scientific Electronic Library Online (SCIELO) e Scopus.

Os critérios de inclusão para composição do corpus de análise consistiram em: seleção dos artigos com textos disponíveis na íntegra, no idioma inglês ou português, sem limite de data de início da publicação até 18 de julho de 2018. Por sua vez, foram excluídos artigos duplicados e do tipo relato de caso, reflexões e editoriais. O fluxo de seleção dos artigos está representado na Figura 1. 


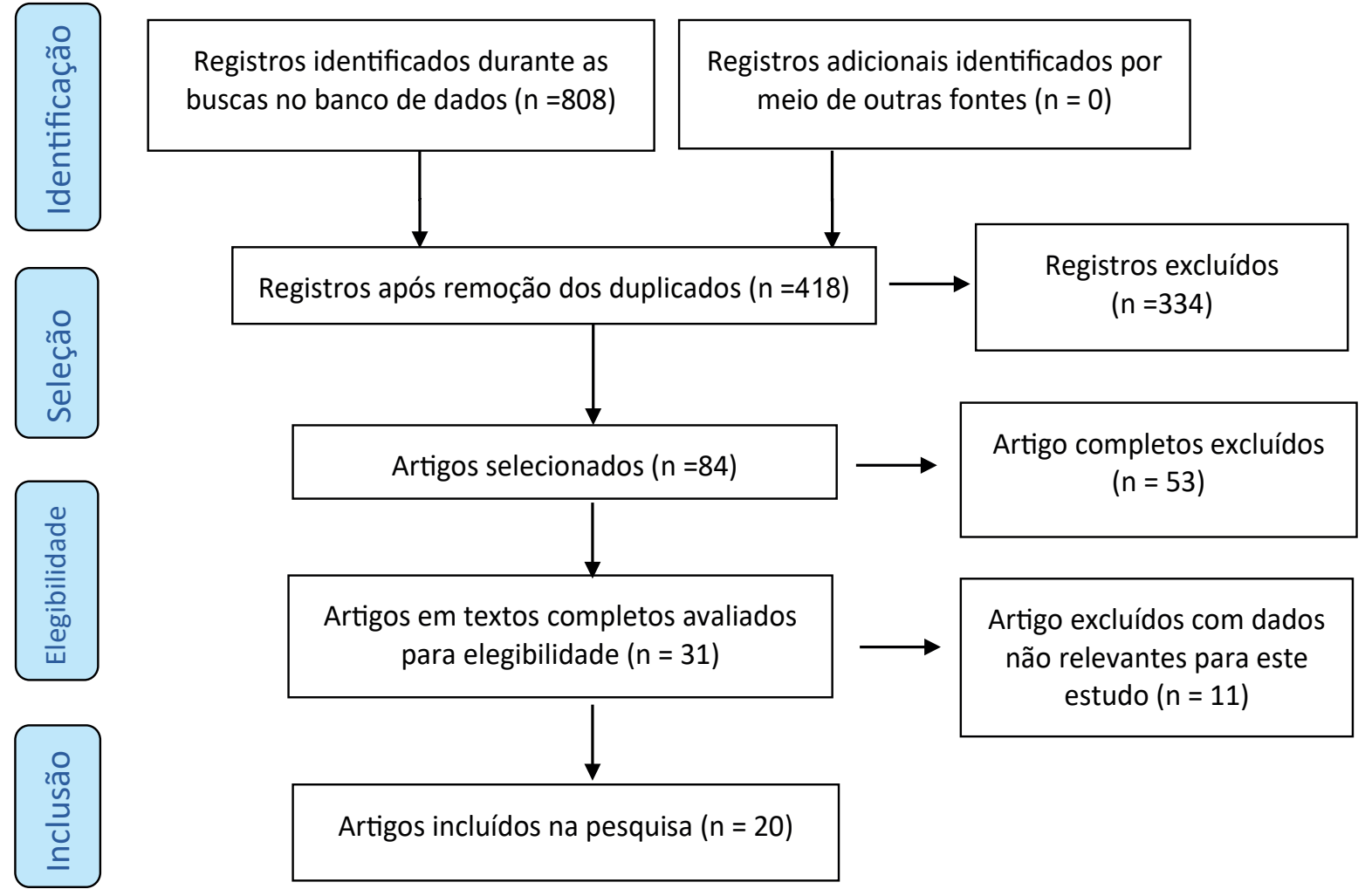

Figura 1 - Procedimento para seleção dos artigos ${ }^{13}$.

Fonte: Adaptado de Galvão TF et al. Principais itens para relatar revisões sistemáticas e meta-análises: a recomendação PRISMA. Epidemiol Serv Saúde. 2015; 24(2): 335-342 ${ }^{13}$.

A seleção dos artigos foi realizada por dois pesquisadores independentes. Incialmente foram excluídos os artigos duplicados. Após, foram aplicados os critérios de inclusão e exclusão propostos ao estudo e na sequência, iniciou-se a seleção para elegibilidade dos artigos que se baseou na avaliação inicial dos títulos e resumos. Por fim, foi realizada análise dos textos completos e suas listas de referências. Esta, para identificar estudos com potencial relevância, não encontrados na busca eletrônica.

Os textos elegíveis foram aqueles que abordavam sobre evidências dos efeitos de Bundles de cuidados na prevenção da PAV em UTI e estudos com Grau de recomendação A e B, classificados de acordo com os critérios estabelecidos pela Oxford Centre for Evidence-based medicine ${ }^{16}$ (revisão sistemática de ensaio clínico randomizado; revisão sistemática de estudo de coorte ou de caso-controle; ensaio clínico controlado randomizado; ensaio clínico bem delineado sem randomização; estudos de caso-controle; estudo de coorte retrospectivo, contemporâneo ou prospectivo; estudo ecológico). Possíveis discordâncias durante o processo de seleção foram resolvidas consensualmente, entre os pesquisadores. Este consenso foi realizado por meio de respostas que se enquadravam ao objetivo do estudo e também, quando atingia os critérios estabelecidos.

Para organização dos resultados primários, extraiu-se dos textos eleitos, informações que caracterizavam os estudos, como: Referência, ano de publicação e país de realização do estudo; Objetivo(s); Grau de recomendação científica e tipos de estudo; Intervenção para prevenção de PAV (bundle ou bundle associado à outras ações preventivas) e conformidade com a intervenção (conformidade/adesão geral e/ou entre itens específicos do bundle); Incidência de PAV antes e depois do bundle; e Conclusão/Conclusões. 


\section{Resultados}

Foram selecionados 84 estudos para leitura na íntegra. Destes, 31 foram considerados elegíveis, mas 11 não apresentavam dados que respondessem à pergunta da pesquisa. Ao final, 20 artigos compuseram a amostra.

No Quadro 2 constam informações referentes às 20 publicações analisadas nesta revisão.

\section{Quadro 2. Características dos estudos incluídos na revisão. Brasil, 2019}

\begin{tabular}{|c|c|c|c|c|c|}
\hline $\begin{array}{l}\text { Referência } \\
\text { /ano/país }\end{array}$ & Objetivo(s) & $\begin{array}{l}\text { GR* / Tipo de } \\
\text { Estudo }\end{array}$ & $\begin{array}{l}\text { Intervenção (I) / } \\
\text { Conformidade (C) }\end{array}$ & $\begin{array}{l}\text { Principais Resultados } \\
\text { (Efeitos do Bundle) }\end{array}$ & Conclusões \\
\hline $\begin{array}{l}17 / 2013 \\
\text { EUA }\end{array}$ & $\begin{array}{l}\text { Analisar tendências } \\
\text { temporais da } \\
\text { incidência da PAV e o } \\
\text { efeito do bundle }\end{array}$ & $\begin{array}{l}\text { B / Estudo de } \\
\text { coorte } \\
\text { retrospectivo }\end{array}$ & $\begin{array}{l}\text { I: Bundle } \\
\text { C: } 97 \%\end{array}$ & $\begin{array}{c}\text { Pré bundle: variou entre 7,1 } \\
\text { a 10,4/1000 VM-dia } \\
\text { Pós bundle: 10,1/1000 VM-dia }\end{array}$ & $\begin{array}{c}\text { A evidência de que o bundle } \\
\text { previne a PAV é controversa; a } \\
\text { taxa não alterou. }\end{array}$ \\
\hline $\begin{array}{l}18 / 2014 \\
\text { Coreia } \\
\text { do Sul }\end{array}$ & $\begin{array}{l}\text { Investigar a eficácia } \\
\text { de um bundle na } \\
\text { prevenção da PAV }\end{array}$ & $\begin{array}{l}\text { B / Estudo } \\
\text { quase } \\
\text { experimental }\end{array}$ & $\begin{array}{c}\text { I: Bundle } \\
\text { C: pré } 41,1 \% \text { e } \\
\text { pós } 71,8 \%\end{array}$ & $\begin{array}{l}\text { Pré bundle: 4,08/1000 VM-dia } \\
\text { Pós bundle: 1,16/1000 VM-dia }\end{array}$ & $\begin{array}{c}\text { A aplicação adequada do } \\
\text { pacote PAV reduziu } \\
\text { significativamente a incidência } \\
\text { de PAV. }\end{array}$ \\
\hline $\begin{array}{l}19 / 2012 \\
\text { China }\end{array}$ & $\begin{array}{l}\text { Analisar o impacto de } \\
\text { uma abordagem } \\
\text { multidimensional nas } \\
\text { taxas de PAV em UTI }\end{array}$ & $\begin{array}{l}\text { B / Vigilância } \\
\text { prospectiva }\end{array}$ & $\begin{array}{l}\text { I: Bundle/Abor- } \\
\text { dagem multidi- } \\
\text { mensional } \\
\text { C: Cumprimento } \\
\text { quase perfeito }\end{array}$ & $\begin{array}{l}\text { Pré bundle: 24,1/1000 VM-dia } \\
\text { Pós bundle: 5,7/1000 VM-dia } \\
\text { Redução na taxa de PAV (79\%) }\end{array}$ & $\begin{array}{l}\text { Houve redução cumulativa } \\
\text { significativa na taxa de PAV. }\end{array}$ \\
\hline $\begin{array}{l}20 / 2013 \\
\text { Cuba }\end{array}$ & $\begin{array}{l}\text { Avaliar o efeito da } \\
\text { abordagem } \\
\text { multidimensional nas } \\
\text { taxas de PAV em UTI } \\
\text { adulto }\end{array}$ & $\begin{array}{l}\text { B / Estudo } \\
\text { coorte } \\
\text { prospectivo }\end{array}$ & $\begin{array}{l}\text { I: Bundle/Abor- } \\
\text { dagem multidi- } \\
\text { mensional } \\
\text { C: } 56 \%\end{array}$ & $\begin{array}{l}\text { Pré bundle: 52,63/1000 VM-dia } \\
\text { Pós bundle: } 15,32 / 1000 \text { VM-dia } \\
\text { Redução cumulativa } \\
\text { significativa na taxa de PAV } \\
\text { ( } 70 \% \text { nos } 3 \text { anos) }\end{array}$ & $\begin{array}{c}\text { A abordagem } \\
\text { multidimensional se associou } \\
\text { com a redução significativa na } \\
\text { taxa da PAV. }\end{array}$ \\
\hline $\begin{array}{c}21 / 2015 \\
\text { Brasil }\end{array}$ & $\begin{array}{l}\text { Analisar o efeito do } \\
\text { bundle nas taxas de } \\
\text { PAV em UTI pediátrica }\end{array}$ & $\begin{array}{l}\text { B / Estudo } \\
\text { transversal }\end{array}$ & $\begin{array}{c}\text { I: Bundle } \\
\text { C: pré } 61 \% \text { a } \\
100 \% \text { e pós } 37 \% \\
\text { a } 100 \% \text { entre itens }\end{array}$ & $\begin{array}{c}\text { Pré bundle: } 49,6 \% \\
\text { Pós bundle: } 17,5 \% \\
\text { Redução significativa na } \\
\text { taxa de PAV }(64,8 \%)\end{array}$ & $\begin{array}{c}\text { O uso do bundle se associou } \\
\text { com a redução significativa na } \\
\text { taxa de PAV. }\end{array}$ \\
\hline $\begin{array}{c}\text { 22/2016 } \\
\text { Brasil }\end{array}$ & $\begin{array}{c}\text { Avaliar impacto e } \\
\text { fatores determinantes } \\
\text { no cumprimento do } \\
\text { bundle para redução } \\
\text { da PAV }\end{array}$ & $\begin{array}{l}\text { B/Estudo } \\
\text { longitudinal } \\
\text { retrospectivo }\end{array}$ & $\begin{array}{c}\text { I: Bundle } \\
\text { C: Baixa adesão }\end{array}$ & $\begin{array}{l}\text { Pré bundle: 11,53/1000 VM-dia } \\
\text { Pós bundle: 16,42/1000 VM-dia }\end{array}$ & $\begin{array}{l}\text { Houve aumento na incidência } \\
\text { de PAV e, baixa adesão ao } \\
\text { cumprimento do bundle }\end{array}$ \\
\hline $\begin{array}{l}23 / 2017 \\
\text { EUA }\end{array}$ & $\begin{array}{c}\text { Avaliar o efeito do } \\
\text { bundle nas taxas de } \\
\text { PAV }\end{array}$ & $\begin{array}{l}\text { B / Estudo } \\
\text { antes e } \\
\text { depois }\end{array}$ & $\begin{array}{l}\text { I: Bundle } \\
\text { C: } 6 \% \text { a } 89 \% \\
\text { entre itens }\end{array}$ & $\begin{array}{c}\text { Pré bundle: } 11,3 \% \\
\text { Pós bundle: 5,7\%; e 3,9\% no } \\
\text { DE (Departamento de } \\
\text { Emergência) }\end{array}$ & $\begin{array}{c}\text { Redução significativa na taxa } \\
\text { de PAV }\end{array}$ \\
\hline $\begin{array}{l}\text { 24/2018 } \\
\text { Espanha }\end{array}$ & $\begin{array}{l}\text { Avaliar o impacto do } \\
\text { projeto "Pneumonia } \\
\text { Zero" na redução das } \\
\text { taxas de PAV em UTIs } \\
\text { espanholas }\end{array}$ & $\begin{array}{c}\text { B / Estudo } \\
\text { prospectivo, } \\
\text { intervencionista }\end{array}$ & $\begin{array}{c}\text { I: Bundle - } \\
\text { Pneumonia Zero } \\
\text { C: } 62 \% \text { geral e } \\
9,4 \% \text { a } 60,2 \% \\
\text { entre itens }\end{array}$ & $\begin{array}{l}\text { Pré bundle: 9,83/1000 VM-dia } \\
\text { Pós bundle: 4,34/1000 VM-dia } \\
\text { Redução de 2,4\% para 1,9\% }\end{array}$ & $\begin{array}{c}\text { Redução significativa da } \\
\text { incidência de PAV em UTIs } \\
\text { espanholas. }\end{array}$ \\
\hline $\begin{array}{l}25 / 2016 \\
\text { Turquia }\end{array}$ & $\begin{array}{c}\text { Investigar o efeito do } \\
\text { pacote assistencial } \\
\text { nas taxas de PAV }\end{array}$ & $\begin{array}{l}\text { B / Estudo } \\
\text { quase- } \\
\text { experimental }\end{array}$ & $\begin{array}{c}\text { I: Bundle } \\
\text { C: pré } 10,8 \% \text { e } \\
\text { pós } 89,8 \%\end{array}$ & $\begin{array}{l}\text { Pré bundle: 15,91/1000 VM-dia } \\
\text { Pós bundle: 8,50/1000 VM-dia }\end{array}$ & $\begin{array}{l}\text { O uso do bundle se associou } \\
\text { com a redução na taxa de PAV. }\end{array}$ \\
\hline $\begin{array}{l}26 / 2015 \\
\text { Taiwan }\end{array}$ & $\begin{array}{c}\text { Implementar um } \\
\text { bundle de PAV em } \\
\text { cinco UTIs e avaliar a } \\
\text { sua eficácia na } \\
\text { redução da densidade } \\
\text { de PAV }\end{array}$ & $\begin{array}{l}\text { B / Estudo } \\
\text { transversal }\end{array}$ & $\begin{array}{c}\text { I: } \text { Bundle } \\
\text { C: } 97,9 \%, 80.3 \% \text {, } \\
\text { e } 73.7 \% \text { para } \\
\text { médicos, } \\
\text { enfermeiros e } \\
\text { fisioterapeutas } \\
\text { respectivamente. }\end{array}$ & $\begin{array}{c}\text { Pré bundle: } 3,3 / 1000 \text { VM-dia } \\
\text { Pós bundle: 1,4/1000 VM-dia } \\
\text { - Incidência de 13,6 para } \\
\text { 3,9/1000 VM-dia } \\
\text { Redução de 1,9/1000 VM- } \\
\text { dia }(57,6 \%) \text {. }\end{array}$ & $\begin{array}{c}\text { O bundle foi eficaz na redução } \\
\text { da incidência de PAV, no uso } \\
\text { do ventilador e densidade de } \\
\text { PAV. }\end{array}$ \\
\hline $\begin{array}{l}\text { 27/2016 } \\
\text { Grécia }\end{array}$ & $\begin{array}{l}\text { Examinar os efeitos } \\
\text { da implementação de } \\
\text { bundle na incidência } \\
\text { de PAV na UTI }\end{array}$ & $\begin{array}{l}\mathrm{B} / \text { Prospectivo } \\
\text { intervencionista }\end{array}$ & $\begin{array}{c}\text { I: Bundle } \\
\text { C: pré } 13 \% \text { e pós } \\
28 \%\end{array}$ & $\begin{array}{l}\text { Pré bundle: 21,6//1000 VM-dia } \\
\text { Pós bundle: 11,6//1000 VM-dia }\end{array}$ & $\begin{array}{l}\text { Houve redução na incidência } \\
\text { de PAV após implantação do } \\
\text { bundle. }\end{array}$ \\
\hline
\end{tabular}




\begin{tabular}{|c|c|c|c|c|c|}
\hline $\begin{array}{l}\text { Referência } \\
\text { /ano/país }\end{array}$ & Objetivo(s) & $\begin{array}{l}\text { GR* / Tipo de } \\
\text { Estudo }\end{array}$ & $\begin{array}{l}\text { Intervenção (I) / } \\
\text { Conformidade (C) }\end{array}$ & $\begin{array}{l}\text { Principais Resultados } \\
\text { (Efeitos do Bundle) }\end{array}$ & Conclusões \\
\hline $\begin{array}{l}\text { 28/2014 } \\
\text { Itália }\end{array}$ & $\begin{array}{c}\text { Relatar o impacto de } \\
\text { um programa } \\
\text { multifacetado na } \\
\text { prevenção de PAV em } \\
\text { UTI }\end{array}$ & $\begin{array}{l}\text { B / Estudo } \\
\text { longitudinal } \\
\text { retrospectivo }\end{array}$ & $\begin{array}{c}\text { I: Bundle } \\
\text { C: não apresenta }\end{array}$ & $\begin{array}{c}\text { Pré bundle: } 15,9 \% \\
\text { Pós bundle: } 6,7 \% \\
\text { Redução na PAV precoce } \\
\text { (6,6\% para 1,9\%) e PAV } \\
\text { tardio ( } 9,3 \% \text { para } 4,7 \%) .\end{array}$ & $\begin{array}{c}\text { A implementação de uma } \\
\text { abordagem multifacetada se } \\
\text { associou com a redução } \\
\text { significativa na ocorrência de } \\
\text { PAV. }\end{array}$ \\
\hline $\begin{array}{l}\text { 29/2016 } \\
\text { Espanha }\end{array}$ & $\begin{array}{c}\text { Avaliar se a } \\
\text { implementação de } \\
\text { um bundle reduz } \\
\text { infecções associadas à } \\
\text { ventilação mecânica } \\
\text { em UTI pediátrica }\end{array}$ & $\begin{array}{l}\text { B / Prospectivo } \\
\text { intervencionista }\end{array}$ & $\begin{array}{c}\text { I: Bundle } \\
\text { C: não apresenta }\end{array}$ & $\begin{array}{c}\text { Pré bundle: - 4,14/1000 VM- } \\
\text { dia; paciente com } \\
\text { traqueostomia } 8,16 / / 1000 \\
\text { VM-dia } \\
\text { Pós bundle: - 1,05/1000 VM- } \\
\text { dia; traqueostomia } \\
\text { 0,65/1000 VM-dia } \\
\text { PAV diminuiu 74,7\% }\end{array}$ & $\begin{array}{c}\text { A implementação de um } \\
\text { bundle diminuiu a incidência } \\
\text { de PAV e atrasou o início da } \\
\text { traqueobronquite associada à } \\
\text { VM em crianças. }\end{array}$ \\
\hline $\begin{array}{l}\text { 30/2014 } \\
\text { Arábia } \\
\text { Saudita }\end{array}$ & $\begin{array}{l}\text { Avaliar a associação } \\
\text { do bundle com a taxa } \\
\text { de PAV em um } \\
\text { ambiente com alta } \\
\text { incidência de PAV }\end{array}$ & $\begin{array}{l}\text { B /Estudo } \\
\text { Transversal }\end{array}$ & $\begin{array}{c}\text { I: Bundle } \\
\text { C: } 90 \%(2010) \text { e } \\
97 \%(2013)\end{array}$ & $\begin{array}{c}\text { Pré bundle: 3,6/1000 VM-dia } \\
\text { Pós bundle: 1,0/1000 VM-dia } \\
\text { Melhora de } 70 \% \text { nas taxas } \\
\text { de PAV }\end{array}$ & $\begin{array}{l}\text { Foram observadas melhora } \\
\text { nas taxas de PAV durante a } \\
\text { implementação do bundle. }\end{array}$ \\
\hline $\begin{array}{l}31 / 2017 \\
\text { Egito }\end{array}$ & $\begin{array}{l}\text { Avaliar a adesão ao } \\
\text { bundle e seu efeito } \\
\text { nas taxas de PAV, } \\
\text { mortalidade e custos } \\
\text { com a assistência }\end{array}$ & $\begin{array}{l}\text { B / Prospectivo } \\
\text { Intervencionista }\end{array}$ & $\begin{array}{c}\text { I: Bundle } \\
\text { C: } 94 \% \text { a } 100 \%\end{array}$ & $\begin{array}{c}\text { Pré bundle: } 18,5 \% ; 25 / 1000 \\
\text { VM-dia } \\
\text { Pós bundle: } 9 \% ; 8,5 / 1000 \\
\text { VM-dia (2014) e 6/1000 VM- } \\
\text { dia (2015) }\end{array}$ & $\begin{array}{l}\text { O bundle produziu melhora } \\
\text { nos índices de PAV, com } \\
\text { redução da mortalidade, } \\
\text { tempo de internação e custos } \\
\text { com assistência }\end{array}$ \\
\hline $\begin{array}{c}32 / 2016 \\
\text { Brasil }\end{array}$ & $\begin{array}{c}\text { Avaliar o impacto de } \\
\text { um pacote FAST HUG } \\
\text { na incidência da PAV, } \\
\text { custos, mortalidade e } \\
\text { tempo de } \\
\text { permanência do } \\
\text { paciente na UTI }\end{array}$ & $\begin{array}{l}\text { B /Estudo } \\
\text { Transversal }\end{array}$ & $\begin{array}{l}\text { I: Bundle - FAST } \\
\text { HUG } \\
\text { C: não apresenta }\end{array}$ & $\begin{array}{l}\text { Pré bundle: } 26 \% \\
\text { Pós bundle: } 9,6 \%\end{array}$ & $\begin{array}{l}\text { A implantação do FAST HUG } \\
\text { reduziu o número de casos de } \\
\text { PAV, os custos, da mortalidade } \\
\text { e o tempo de permanência do } \\
\text { paciente. }\end{array}$ \\
\hline $\begin{array}{c}33 / 2012 \\
\text { EUA }\end{array}$ & $\begin{array}{l}\text { Desenvolver bundle e } \\
\text { práticas de cuidados } \\
\text { de enfermagem para } \\
\text { prevenção de PAV em } \\
\text { UTI }\end{array}$ & $\begin{array}{l}\text { B /Estudo } \\
\text { Prospectivo }\end{array}$ & $\begin{array}{l}\text { I: Bundle } \\
\text { C: } 76 \% \text { a } 100 \% \\
\text { entre itens }\end{array}$ & $\begin{array}{l}\text { Pré bundle: 9,47/1000 VM-dia } \\
\text { Pós bundle: 1,9/1000 VM-dia }\end{array}$ & $\begin{array}{l}\text { A adesão estrita ao bundle } \\
\text { pela equipe multidisciplinar } \\
\text { resultou em uma redução } \\
\text { significativa nas taxas de PAV. }\end{array}$ \\
\hline $\begin{array}{l}34 / 2010 \\
\text { EUA }\end{array}$ & $\begin{array}{l}\text { Examinar o impacto } \\
\text { da adesão de um } \\
\text { bundle na incidência } \\
\text { de PAV em duas UTIs }\end{array}$ & $\begin{array}{l}\text { B / Estudo } \\
\text { Transversal }\end{array}$ & $\begin{array}{c}\text { I: Bundle } \\
\text { C: } 53 \% \text { para } 91 \% \\
\text { (UTI A) e; } 63 \% \\
\text { para } 81 \% \text { (UTI B) }\end{array}$ & $\begin{array}{l}\text { Pré bundle: 10,2/1000 VM-dia } \\
\text { Pós bundle: 3,4/1000 VM-dia } \\
\text { Redução do risco: } 67 \%\end{array}$ & $\begin{array}{l}\text { O bundle se associou com a } \\
\text { redução da incidência de PAV } \\
\text { e custos hospitalares. }\end{array}$ \\
\hline $\begin{array}{l}35 / 2015 \\
\text { Egito }\end{array}$ & $\begin{array}{c}\text { Avaliar a eficácia do } \\
\text { bundle na redução da } \\
\text { taxa de PAV em UTI } \\
\text { neonatal }\end{array}$ & $\begin{array}{l}\text { B / Coorte } \\
\text { intervencionista }\end{array}$ & $\begin{array}{c}\text { I: Bundle } \\
\text { C: não apresenta }\end{array}$ & $\begin{array}{c}\text { Pré bundle: } 36,4 / 1000 \text { VM- } \\
\text { dia; } 67,8 \% \\
\text { Pós bundle: } 23 / 1000 \text { VM- } \\
\text { dia; } 38,2 \%\end{array}$ & $\begin{array}{c}\text { A implementação do bundle se } \\
\text { associou com a redução na } \\
\text { taxa de PAV na UTI neonatal. }\end{array}$ \\
\hline $\begin{array}{l}\text { 36/2011 } \\
\text { Escócia }\end{array}$ & $\begin{array}{c}\text { Determinar os efeitos } \\
\text { da implementação de } \\
\text { um bundle na } \\
\text { incidência da PAV }\end{array}$ & $\begin{array}{l}\text { B /Estudo Antes } \\
\text { e depois }\end{array}$ & $\begin{array}{c}\text { I: Bundle } \\
\text { C: } 70 \% \text { geral e } \\
70 \% \text { a } 100 \% \\
\text { entre itens }\end{array}$ & $\begin{array}{l}\text { Pré bundle: 32/1000 VM-dia } \\
\text { Pós bundle: 12/1000 VM-dia }\end{array}$ & $\begin{array}{l}\text { A implementação de um } \\
\text { bundle se associou com a } \\
\text { redução significativa na } \\
\text { incidência da PAV. }\end{array}$ \\
\hline
\end{tabular}

*Grau de recomendação ${ }^{16}$; VM: ventilação mecânica; PAV: pneumonia associada à ventilação mecânica; UTI: unidade de terapia intensiva.

Fonte: Elaboração própria

Os estudos eleitos foram publicados entre 2010 e 2018, com predomínio nos últimos cinco anos (70\%), desenvolvidos nos diferentes continentes, com exceção da Oceania e Antártida. Houve distribuição quase que equitativa entre os continentes referidos, porém ao analisar a frequência dos estudos por país, houve destaque para os Estados Unidos ${ }^{14,20,30-31}$ (20\%), Brasil ${ }^{18,19,29}$ (15\%), Espanha ${ }^{21,26}$ e Egito ${ }^{28,32}$. Estes dois últimos, com $10 \%$. 
Quanto ao grau de recomendação, de acordo com os critérios estipulados pela Oxford Centre for Evidence-based medicine ${ }^{16}$, todos foram classificados com grau de recomendação $\mathrm{B}$, prevalecendo os delineamentos de estudos prospectivos intervencionistas e longitudinais retrospectivos, ambos $35 \%$.

No que tange a unidade investigada, a maioria (85\%) dos estudos implementou o bundle de prevenção de PAV em UTIs adultos, dois ${ }^{18,38}(10 \%)$ em UTIs pediátricas e apenas um ${ }^{32}$ (5\%) em UTI neonatal.

A maioria dos estudos $(\mathrm{n}=18 ; 90 \%)^{18-21,23-36}$ revelou que a aplicabilidade de um bundle de cuidados na prevenção da PAV reduziu a incidência da PAV, com impacto na redução da mortalidade, no tempo de internação e nos custos hospitalares. No entanto, um (5\%) estudo ${ }^{17}$ aborda que não foi observada diminuição na densidade da incidência da PAV em UTI e outro $(1=5 \%)^{22}$ constatou aumento nas taxas de PAV após implementação do bundle.

Referente aos itens de cuidados que constam no bundle, não foi constatado padronização na quantidade de componentes individuais de assistência na prevenção

A maioria dos estudos $(n=18 ; 90 \%)$ revelou que a aplicabilidade de um bundle de cuidados na prevenção da PAV reduziu a incidência da PAV, com impacto na redução da mortalidade, no tempo de internação e nos custos hospitalares. da PAV nos estudos, pois se observou mínimo de quatro e máximo de 14 itens. Apesar da versatilidade nos itens de cuidados, foi observada semelhança nos bundles destinados aos adultos, crianças e neonatos. Os 10 cuidados prevalentes nos bundles de prevenção da PAV estão descritos na Tabela 1.

Tabela 1. Frequência de cuidados inseridos nos Bundles para prevenção de PAV em UTI. Brasil, 2019.

\begin{tabular}{lcc}
\hline \multicolumn{1}{c}{ Cuidados } & n & $\%$ \\
\hline Elevação da cabeceira & 20 & 100 \\
Protocolo de higiene oral & 17 & 85 \\
Interrupção diária da sedação & 13 & 65 \\
Avaliação diária da possibilidade de extubação & 13 & 65 \\
Profilaxia para trombose venosa profunda & 11 & 55 \\
Profilaxia para úlcera péptica/de estresse & 10 & 50 \\
Controle da pressão do cuff & 9 & 45 \\
Higiene das mãos & 8 & 40 \\
Aspiração da secreção supraglótica & 6 & 30 \\
Troca de extensões úmidas ou condensadas & 6 & 30 \\
\hline
\end{tabular}

Fonte: dados do estudo.

Dos 20 estudos primários incluídos, apenas 12 (60\%) 17-20,24-27,30,31,34,36 apresentaram o índice de conformidade geral com o bundle, com valores que variaram entre $28 \%^{27}$ a $100 \%{ }^{31}$. Sete (35\%) estudos ${ }^{18,21,23,24,33,34,36}$ apresentaram a conformidade com itens específicos do bundle e, dentre estes, os menores índices de adesão se referiam aos cuidados com a interrupção diária da sedação/testes de respiração espontânea ${ }^{23}(6 \%)$ e; aspiração contínua de secreção subglótica ${ }^{24}$ (9,4\%). Por sua vez, os maiores índices foram para higiene das mãos, decúbito do leito elevado $30^{\circ}$ 
a 45, profilaxia para úlcera péptica, profilaxia para trombose venosa profunda (TVP), interrupção diária da sedação e; avaliação para extubação (100\%) ${ }^{21,33}$. Cinco (25\%) estudos 22,28,29,32,35 não delinearam a conformidade do pacote de cuidados.

Um (5\%) estudo ${ }^{26}$ verificou as taxas gerais de conformidade entre a equipe multiprofissional e constatou que o melhor índice de cumprimento ao bundle se referia ao médico $(97,7 \%)$, seguido pelo enfermeiro $(80,3 \%)$ e fisioterapeuta $(73,7 \%)$. Já outro estudo, realizado em unidade de terapia intensiva pediátrica no Brasil $^{21}$ constatou melhores índices de adesão a alguns elementos do pacote de cuidados por enfermeiros e fisioterapeutas (100\%), seguido pelos médicos (98\%).

\section{Discussão}

A incidência da PAV pode estar relacionada a falhas no processo de cuidado, o que oportuniza a ocorrência de complicações na condição de saúde dos pacientes hospitalizados e, repercute em aumento do tempo de internação e nos custos hospitalares ${ }^{3}$. A estimativa da mortalidade atribuída a essa infecção pode variar, mas aproximadamente 33\% dos pacientes com PAV morrem em decorrência direta desta infecção e a mortalidade global, oscila entre 20 a 60\%, cuja grande parte ocorre em virtude da severidade da doença de base e, especificidades da população estudada ${ }^{3,22}$.

Dentre os estudos analisados, foi possível constatar que, na maioria 18-21,23-36, a aplicabilidade de um bundle de prevenção da PAV em UTI reduziu a incidência desta afecção e impactou positivamente na mortalidade, no tempo de internação e nos custos assistenciais. Por outro lado, um estudo apresentou a manutenção ${ }^{17}$ da taxa e, outro constatou até mesmo o aumento ${ }^{22}$ da densidade da PAV, após a implementação do pacote de cuidados, reforçando a importância do monitoramento e da reavaliação/replanejamento contínuo, no uso de bundles $26,031,37$.

Existem variáveis que atuam de forma direta ou indireta no aumento da incidência da PAV após a implementação do bundle como por exemplo, baixa adesão ao cumprimento do pacote de cuidados pelos profissionais, ausência ou insuficiência de programas educacionais e de suporte à sua implementação e; déficit no suprimento de insumos assistenciais ${ }^{22}$. Em contrapartida, a manutenção da taxa de PAV, verificada em um dos estudos ${ }^{17}$, pode ser atribuída à alta adesão aos elementos de cuidados já observados na UTI antes mesmo da implementação do bundle, justificando índices semelhantes de PAV nos períodos pré e pós implementação do pacote de cuidados $^{17}$.

Observou-se que a aplicabilidade do bundle de prevenção da PAV em UTI reduziu a incidência do evento e da mortalidade ${ }^{31,32}$. Entretanto, consta que fatores específicos podem influenciar nestes resultados, tais como: idade, procedência, uso indiscriminado de antibióticos, inadequada higienização oral dos pacientes e das mãos de qualquer pessoa que tenha contato com o indivíduo hospitalizado ${ }^{22}$. Neste sentido, estudos ${ }^{22,26}$ recomendam alerta para os fatores que podem interferir nas taxas de PAV, a fim de sensibilizar a equipe multidisciplinar às boas práticas e assim, garantir a sistematização das intervenções preventivas ${ }^{18,19}$.

No que diz respeito aos efeitos positivos da aplicabilidade de bundle e outras medidas preventivas, um estudo na China $^{19}$, relatou resultados positivos na implementação de uma intervenção 
multidimensional para o controle de infecção da PAV em UTI, pois observou redução significativa na taxa desse evento adverso. A abordagem incluiu a educação e treinamento para a equipe multiprofissional, promoção da adesão a diretrizes preventivas, feedback das taxas de PAV para a equipe de saúde, vigilância do processo, uso de ferramentas de observação estruturadas em intervalos regularmente programados e feedback de desempenho das práticas de controle de infecção ${ }^{19}$.

O estudo supracitado concluiu que a aplicação de uma única medida de controle de infecção não é suficiente para prevenir a PAV, pois isso requer mudanças na cultura de trabalho e no envolvimento de toda a equipe de profissionais de saúde ${ }^{19}$. Essa assertiva vem ao encontro da Agência Nacional de Vigilância Sanitária (ANVISA) ${ }^{3}$ do Brasil e de outros estudos ${ }^{20,26,28,33}$ que afirmam que a cultura organizacional, o trabalho em equipe e o treinamento da equipe multiprofissional que presta assistência aos pacientes em ventilação mecânica é fundamental e que isso, tem impacto direto nas taxas de PAV. Além disso, afirmam que as estratégias educativas com enfoque na prevenção da PAV devem, de preferência, serem multimodais, ou seja, envolver metodologias variadas como, treinamento por meio de aula presencial, e-learning, aula prática e com simulações, discussão da prática à beira do leito e; feedback de resultados de indicadores, com discussão de medidas preventivas para os profissionais $20,26,28,33$.

Estudos ${ }^{21,26}$ relatam que o pacote de cuidados é uma medida eficaz para reduzir a incidência de PAV na UTI e o cumprimento do pacote no trabalho em equipe multidisciplinar, a educação continuada e o planejamento de uma lista de verificação abrangente para melhorar a conformidade dos profissionais de saúde são essenciais para que isso aconteça. Logo, para o cumprimento destes pacotes de cuidados, elementos como conhecimento, habilidades, atitudes e responsabilidade no cuidar de forma conjunta por parte da equipe, pode proporcionar a prevenção da PAV, melhorias no tratamento e na O pacote de cuidados é uma medida eficaz para reduzir a incidência de PAV na UTI e o cumprimento do pacote no trabalho em equipe multidisciplinar, a educação continuada e o planejamento de uma lista de verificação abrangente para melhorar a conformidade dos profissionais de saúde são essenciais para que isso aconteça. recuperação do paciente/cliente ${ }^{37}$.

No que tange ao cumprimento adequado do bundle, dentre os estudos analisados, constatouse variação discrepante no índice de conformidade geral ${ }^{17,27,31}$ e de elementos individuais do pacote de cuidados ${ }^{21,23,24}$. A adesão elevada a alguns itens específicos do bundle refletiu em resultados positivos, como por exemplo, a descontaminação oral com clorexidina 0,12\%, que foi destacada como o item com o maior impacto na redução da PAV, mas ainda assim os autores reforçam que mais evidências são necessárias para que isto realmente seja comprovado ${ }^{18}$. Por sua vez, a clorexidina é classificada como medida de efeito moderado, mas que ao ser aplicada em conjunto com outras medidas, pode ter efeito positivo na redução da PAV ${ }^{38,39}$ e isto corrobora com a necessidade de atenção especial à higiene oral do paciente, quando se pensa em cuidados à saúde.

No que se refere à adesão ao bundle em UTI, estudos desenvolvidos no Brasil ${ }^{21}$, Espanha ${ }^{24}$, Turquia $^{25}$, Taiwan ${ }^{26}$ e nos Estados Unidos ${ }^{33}$, constataram aumento no seu uso, evidenciando melhorias nas práticas de higienização das mãos, profilaxia de úlcera péptica e trombose venosa profunda, interrupção da sedação, elevação da cabeceira do leito, remoção de líquido condensado no circuito do ventilador mecânico, pressão do cuff endotraqueal, dentre outros. Vale ressaltar que essas práticas são indicadas como medidas preventivas da PAV, porém com diferentes níveis de evidências, classificadas desde fortemente recomendada por ser apoiada em 
estudos experimentais, clínicos ou epidemiológicos (IA), até nenhuma recomendação/questão não resolvida (NR), por não existir evidência suficiente ou consenso sobre a sua eficácia ${ }^{8}$.

Neste cenário multicêntrico, apesar de diferentes características demográficas, políticas, sociais e econômicas, peculiares entre os países abordados na presente pesquisa, nota-se que há um senso comum acerca da implementação de estratégias eficazes para aplicação de pacotes de cuidados na PAV e utilização de protocolos baseados em evidências no atendimento em UTI, como o que é preconizado pelo Institute for Healthcare Improvement ${ }^{38}$. No entanto, inobservou estandartização da seleção dos principais componentes e da quantidade destes elementos de cuidados nos bundles. A elevação da cabeceira foi a estratégia de cuidado que predominou $(100 \%)^{17-36}$, seguido do protocolo de higiene oral $(85 \%)^{18-33,35,36}$, ambos apoiados em estudos clínicos ou epidemiológicos, sugestivos ou por uma forte fundamentação teórica, conforme preconiza o Centers for Desease Control and Prevention (CDC $)^{8}$.

Para a melhoria na segurança e na qualidade da assistência de pacientes hospitalizados, os bundles têm sido recomendados para substituir as medidas isoladas de prevenção da PAV, mas para isto, são necessários treinamentos e adesão da equipe multidisciplinar de saúde para que possam ser considerados indicadores de qualidade ${ }^{37}$. Uma estratégia que pode ser eficaz no envolvimento dos profissionais ao pacote de cuidados, é o desenvolvimento de oficinas de reeducação, utilização de pôsteres informativos e; até mesmo, a padronização de intervenções e de equipamentos ${ }^{26}$.

Um estudo com objetivo de identificar o conhecimento da equipe de enfermagem acerca da segurança do paciente relacionada ao bundle de prevenção da PAV, aborda à necessidade de conhecimento especifico do profissional para atender a essa demanda de cuidados peculiares ${ }^{40}$. A fragilidade no conhecimento sobre prevenção da PAV remete a necessidade de inclusão do tema segurança do paciente ao longo de todo o processo de formação dos profissionais, sendo essencial a revisão dos projetos político pedagógicos dos cursos de graduação em saúde, bem como a implementação de estratégias que possibilitem o alinhamento desse tema junto às demais disciplinas da área de saúde $\mathrm{e}^{41,42}$.

A PAV, embora seja uma infecção, pode ser evitada pelo cuidado profissional fundamentado nas melhores evidências científicas ${ }^{43}$. Deste modo, é imperativa a convergência de estudos que demonstrem os benefícios ou não de bundles ou outras tecnologias, na prevenção dessa infecção nosocomial, pois o conhecimento, se comprovado e consolidado, poderá subsidiar tomadas de decisões assertivas seja no campo da prática, como do ensino e da pesquisa.

Mediante ao exposto, reitera-se importância das ações deeducação permanente, desenvolvidas junto às equipes, a partir do monitoramento dos indicadores referentes à PAV, específicos a cada localidade. Isso porque, as competências desenvolvidas, oportunizam discussões acerca do processo de trabalho no seu todo, conhecimento das dificuldades vivenciadas na prática rotineira do serviço e o planejamento conjunto de ações que influenciam positivamente na redução das taxas de PAV, especialmente, em UTI.

Como limitação deste estudo, assume-se que o nível de evidência científica por tipo de publicação encontrado nas bases de dados, pois não foi localizado nenhum estudo classificado com grau de recomendação A que representa a melhor força de evidência. Outro fator limitante é a versatilidade e quantidade de elementos de intervenção presente nos pacotes de cuidados, o que compromete o estabelecimento de um padrão que determina a eficácia dos bundles em UTIs adulto, pediátrico e neonatal. Apesar disso, esta revisão contribui por ser um aporte sintético rumo à prática baseada em evidências na prevenção da PAV, o que confere empoderamento aos 
profissionais de saúde no cuidado ao indivíduo ventilado mecanicamente, pois os resultados indicam que a adesão da equipe de saúde, na aplicação de bundles em UTI, nos diferentes cenários mundiais, produz efeitos positivos ao paciente e ao sistema de saúde.

\section{Conclusão}

Apesar de existir espaço para estudos com maior grau de recomendação conclui-se que, o uso do Bundle na prevenção da PAV em UTI tem efeitos positivos, pois estudos desenvolvidos em diferentes países constataram que a adesão a essa ferramenta de segurança resultou em redução na taxa de PAV, na mortalidade de pacientes, no seu tempo de internação e também; nos custos hospitalares. Revelou-se ainda que os principais componentes relacionados a composição do pacote de cuidado foram a elevação da cabeceira e o protocolo de higiene oral, uma vez que todos estudos incluídos nesta revisão, possuem força de evidência classificada como $B$, de acordo com os critérios estabelecidos pela Oxford Centre for Evidence-based medicine.

Conflito de interesses: Os autores declaram que não há conflito de interesses.

Financiamiento: recursos próprios dos autores.

\section{Referências}

1. American Thoracic Society /Infections Diseases Society Of America (ATS /IDSA). Guidelines for the management of adults with hospitalacquired, ventilator-associated, and healthcare-associated pneumonia. Am J Respir Crit Care Med. 2005;171(4):388-416.

https://doi.org/10.1164/rccm.200405-644ST

2. Kollef MH, Chastre J, Fagon JY, François BMD, Niederman MS, et al. Global prospective epidemiologic and surveillance study of ventilator-associated pneumonia due to Pseudomonas aeruginosa. Crit Care Med. 2014;42(10):2178-87.

https://doi.org/10.1097/CCM.0000000000000510

3. Brasil. Agência Nacional de Vigilância Sanitária. Medidas de Prevenção de Infecção Relacionada à Assistência à Saúde. - Brasília: Anvisa, 2017.

4. Mitharwal SM, Yaddanapudi S, Bhardwaj N, Gautam V, Biswal M, Yaddanapudi L. Intensive care unitacquired infections in a tertiary care hospital: An epidemiologic survey and influence on patient outcomes. Am J Infect Control. 2016;44(7):e113-e7. https://doi.org/10.1016/j.ajic.2016.01.021

5. Rahmqvist M, Samuelsson A, Bastami S, Rutberg H. Direct health care costs and length of hospital stay related to health care-acquired infections in adult patients based on point prevalence measurements. Am J Infect Control. 2016;44:500-6. https://doi.org/10.1016/j.ajic.2016.01.035

6. Ego A, Preiser JC, Vincent JL. Impact of diagnostic criteria on the incidence of ventilator associated pneumonia. Chest. 2015;147(2):347-55. https://doi.org/10.1378/chest.14-0610

7. Cardoso VB. Entendimento dos enfermeiros intensivistas sobre as formas de prevenção de pneumonia associada à ventilação mecânica invasiva: uma revisão da literatura. Revista Atualiza Saúde [Internet]. 2015[acesso em 2018 Jun 27];1(1):25-34. Disponível em: http:// atualizarevista.com.br/wp-content/uploads/2015/01/ENTENDIMENTO-DOS-ENFERMEIROS-INTENSIVISTAS-SOBRE-AS-FORMAS-DE-PREVEN\%C3\%87\%C3\%830-DE-PNEUMONIA-ASSOCIADA-A-VENTILA\%C3\%87\%C3\%830-MECANICA-INVASIVA-UMA-REVISAO-DA-LITERATURA-revista-atualiza-saude-v1-n1.pdf 
8. Centers For Disease Control and Prevention - CDC. Guidelines for preventing healthcareassociated pneumonia 2003: recommendations of CDC and the Healthcare Infection Control Practices Advisory Committee- MMWR. [Internet] 2004; [cited 2018 Jun 12]; 53(RR-3): [aprox. 36 telas]. Available from:

https://www.cdc.gov/hicpac/pdf/ guidelines/HApneu2003guidelines.pdf

9. Nascimento TBP, Ramos CH, Silva TS, Torres, VPS. Efetividade das medidas de prevenção e controle de pneumonia associada à ventilação mecânica na UTI. Perspectivas Online Biológicas e Saúde. 2017;7(25):1-24. https://doi.org/10.25242/886872520171136

10.Dixon-Woods M, Pronovost PJ. Patient safety and the problem of many hands. BMJ Qual Saf. 2016;25(7):485-8. https://doi.org/10.1136/bmjqs-2016-005232

11.Araújo MAN, Lunardi Filho WD, Silveira RS, Souza JC, Barlem ELD, Teixeira NS. Segurança do paciente na visão de enfermeiros: uma questão multiprofissional. Enferm. Foco. 2017;8(1):52-6. https://doi.org/10.21675/2357-707X.2017.v8.n1.984

12.Moher D, Liberati A, Tetzlaff J, Altman DG, The PRISMA Group. Preferred Reporting Items for Systematic Reviews and Meta-Analyses: The PRISMA Statement. PLoS Med. 2009; 6(7): e1000097. https://doi.org/10.1371/journal.pmed.1000097.t001

13.Galvão TF, Pansani TDSA, Harrad D. Principais itens para relatar revisões sistemáticas e meta-análises: a recomendação PRISMA. Serv. Saúde. 2015;24(2):335-42. https://doi.org/10.5123/S1679-49742015000200017

14.Whittemore R, Knafl K. The integrative review: updated methodology. J Adv Nurs. 2005;52(5):546-53. https://doi.org/10.1111/j.1365-2648.2005.03621.x

15.Santos CMDC, Pimenta CADM, Nobre MRC. The PICO strategy for the research question construction and evidence search. Rev. Latino-Am. Enfermagem. 2007;15(3):508-11. https://doi.org/10.1590/S0104-11692007000300023

16.0xford Centre for Evidence-based Medicine - Levels of Evidence [Internet] March 2009 [cited 2018 Jul 18] Available from: https://www.cebm.net/2009/06/oxford-centre-evidence-based-medicine-levels-evidence-march-2009/

17.Ding S, Kilickaya O, Senkal S, Gajic O, Hubmayr RD, Li G. Temporal Trends of Ventilator-Associated Pneumonia Incidence and the Effect of Implementing Health-care Bundles in a Suburban Community. Chest. 2013;144(5):1461-8. https://doi.org/10.1378/chest.12-1675

18.Eom JS, Lee MS, Chun HK, Choi HJ, Jung SY, Kim YS, et al. The impact of a ventilator bundle on preventing ventilator-associated pneumonia: A multicenter study. Am J Infect Control. 2014;42(1):34-7. https://doi.org/10.1016/j.ajic.2013.06.023

19.Tao L, Hu B, Rosenthal VD, Zhang Y, Gao X, He L. Impact of a multidimensional approach on ventilator-associated pneumonia rates in a hospital of Shanghai: Findings of the International Nosocomial Infection Control Consortium. J Crit Care. 2012;27(5):440-6.

https://doi.org/10.1016/j.jcrc.2011.12.018

20.Guanche-Garcell H, Morales-Pérez C, Rosenthal VD. Effectiveness of a multidimensional approach for the prevention of ventilator-associated pneumonia in an adult intensive care unit in Cuba: findings of the International Nosocomial Infection Control Consortium (INICC). J Infect Public Health. 2013;6(2):98-107. https://doi.org/10.1016/j.jiph.2012.11.009

21.Perugini MRE, Perugini VH, Figueira FD, Fontana LMS, Diniz JJ, Santos DL, et al. Impacto de um bundle nas taxas de pneumonia associada à ventilação mecânica (PAV) em uma unidade de terapia intensiva pediátrica em Londrina-PR. Semina: Ciênc. Biol. Saúde. 2015;36(1) supl:259-66. http://dx.doi.org/10.5433/1679-0367.2015v36n1Suplp259

22.Rodrigues AN, Fragoso LVC, Beserra FM, Ramos IC. Determining impacts and factors in ventilator-associated pneumonia bundle. Rev Bras Enferm. 2016;69(6):1045-51.

https://doi.org/10.1590/0034-7167-2016-0253

23.DeLuca LA Jr, Walsh P, Davidson DD Jr, Stoneking LR, Yang LM, Grall KJ, et al. Impact and feasibility of an emergency department-based ventilator-associated pneumonia bundle for patients intubated in an academic emergency department. Am J Infect Control. 2017;45(2):151-7. https://doi.org/10.1016/j.ajic.2016.05.037 
24.Álvarez-Lerma F, Palomar-Martínez M, Sánchez-García M, Martínez-Alonso M, Álvarez-Rodríguez J, Lorente $\mathrm{L}$, et al. Prevention of Ventilator-Associated Pneumonia:The Multimodal Approach of the Spanish ICU“Pneumonia Zero"Program. Crit Care Med. 2018;46(2):1818. https://doi.org/10.1097/CCM.0000000000002736

25.Alcan AO, Korkmaz FD, Uyar M. Prevention of ventilator-associated pneumonia: Use of the care bundle approach. Am J Infect Control. 2016;44(10):e173-6.

https://doi.org/10.1016/j.ajic.2016.04.237

26.Lim KP, Kuo SW, Ko WJ, Sheng WH, Chang YY, Hong MC, et al. Efficacy of ventilator-associated pneumonia care bundle for prevention of ventilator-associated pneumonia in the surgical intensive care units of a medical center. J Microbiol Immunol Infect. 2015 48(3):316-21. https://doi.org/10.1016/j.jmii.2013.09.007

27.Parisi M, Gerovasili V, Dimopoulos S, Kampisiouli E, Goga C, Perivolioti E, et al. Use of Ventilator Bundle and Staff Education to Decrease Ventilator-Associated Pneumonia in Intensive Care Patients. Crit Care Nurse. 2016;36(5):e1-e7. https://doi.org/10.4037/ccn2016520

28.Righi E, Aggazzotti G, Ferrari E, Giovanardi C, Busani S, Rinaldi L, et al. Trends in ventilator-associated pneumonia: impact of a ventilator care bundle in an Italian tertiary care hospital intensive care unit. Am J Infect Control. 2014;42(12):1312-6. https://doi.org/10.1016/j.ajic.2014.08.009

29.Peña-López Y, Pujol M, Campins M, González-Antelo A, Rodrigo JÁ, Balcells J, et al. Implementing a care bundle approach reduces ventilator-associated pneumonia and delays ventilator-associated tracheobronchitis in children: differences according to endotracheal or tracheostomy devices. Int JInfect Dis. 2016;52:43-48. https://doi.org/10.1016/j.jijid.2016.09.021

30.Al-Thaqafy MS, El-Saed A, Arabi YM, Balkhy HH. Association of compliance of ventilator bundle with incidence of ventilator-associated pneumonia and ventilator utilization among critical patients over 4 years. Ann Thorac Med. 2014;9(4):221-6.

https://doi.org/10.4103/1817-1737.140132

31.Samra SR, Sherif DM, Elokda SA. Impact of VAP bundle adherence among ventilated critically ill patients and its effectiveness in adult ICU. Egypt J Chest Dis Tuberc. 2017;66(1):81-6. http://dx.doi.org/10.1016/j.ejcdt.2016.08.010

32.Ferreira CR, de Souza DF, Cunha TM, Tavares M, Reis SS, Pedroso RS, et al. The effectiveness of a bundle in the prevention of ventilator-associated pneumonia. Braz J Infect Dis. 2016;20(3):267-71. http://dx.doi.org/10.1016/j.bjid.2016.03.004

33.Sedwick MB, Lance-Smith M, Reeder SJ, Nardi J. Using evidence-based practice to prevent ventilator-associated pneumonia. Crit Care Nurse. 2012;32(4):41-51. https://doi.org/10.4037/ccn2012964

34.Bird D, Zambuto A, O'Donnell C, Silva J, Korn C, Burke R, et al. Adherence to ventilator-associated pneumonia bundle and incidence of ventilator-associated pneumonia in the surgical intensive care unit. Arch Surg. 2010;145(5):465-70.

https://doi.org/10.1001/archsurg.2010.69

35.Azab SF, Sherbiny HS, Saleh SH, Elsaeed WF, Elshafiey MM, Siam AG, et al. Reducing ventilator-associated pneumonia in neonatal intensive care unit using "VAP prevention Bundle": a cohort study. BMC Infect Dis. 2015;15:314-20. https://doi.org/10.1186/s12879-015-1062-1

36.Morris AC, Hay AW, Swann DG, Everingham K, McCulloch C, McNulty J, et al. Reducing ventilator-associated pneumonia in intensive care: impact of implementing a care bundle. Crit Care Med. 2011;39(10):2218-24. https://doi.org/10.1097/CCM.0b013e3182227d52

37.Chicayban LM, Terra ELVS, Ribela JS, \& Barbosa PF. Bundles de prevenção de pneumonia associada à ventilação mecânica: a importância da multidisciplinaridade. Biológicas \& Saúde. 2017;7(25):25-35. https://doi.org/10.25242/886872520171200 
38.Institute for Healthcare Improvement (IHI) - 100,000 Lives Campaign- How-to Guide: Prevent Ventilator-Associated Pneumonia. Cambridge, MA: Institute for Healthcare Improvement; 2012.

https://www.chpso.org/sites/main/files/file-attachments/ihi_howtoguidepreventvap.pdf

39.Klomplas M, Branson R, Eichenwald EC, Greene LR, Howell MD, Lee G, et al. Strategies to Prevent Ventilator- Associated Pneumonia in Acute Care Hospitals: 2014 Update. Infection Control and Hospital Epidemiology 2014;35(S2):S133-54.

https://doi.org/10.1017/S0899823X00193894

40.Tereza DM, Santos MM; Fortunato MM. Relevância da equipe de enfermagem relacionada ao bundle de prevenção de pneumonia associada à ventilação mecânica. Nursing (São Paulo) [Internet] 2017 [acesso em 2018 Jul 18]; 20(232):1816-20. Disponível em: http://bases. bireme.br/cgi-bin/wxislind.exe/iah/online/?IsisScript=iah/iah.xis\&src=google\&base=BDENF\&lang=p\&nextAction=Ink\&exprSearch=31485\&indexSearch=ID

41.Nam-Ju Lee, Haena Jang, Su-Yeon Park. Patient safety education and baccalaureate nursing students' patient safety competency: A cross-sectional study. Nurs. health sci. 2016;1 8:16371. https://doi.org/10.1111/nhs.12237

42. Melleiro MM, Tronchin DMR, Lima MOP, Garzin ACA, Martins MS, Cavalcante MBG, et al. Temática segurança do paciente nas matrizes curriculares de escolas de graduação em enfermagem e obstetrícia. Rev. baiana enferm. 2017;31(2):e16814-21.

http://dx.doi.org/10.18471/rbe.v31i2.16814

43.Maran E, Spigolon DN, Melo WA, Barreto MS, Tostes MFP, Teston EF. Prevenção da pneumonia associada à ventilação mecânica sob a ótica de acadêmicos de enfermagem. Rev Fun Care Online. 2019;11(1):118-123. http://dx.doi.org/10.9789/2175-5361.2019.v11i1.118-123 